\title{
CMOS R-MOSFET-C Fourth-order Bessel Filter with Accurate Group Delay
}

\author{
Yigang He, Jinguang Jiang, Yichuang Sun* \\ College of Electrical and Information Engineering, Hunan University, China, Email: yghe(a)hunu.edu.cn \\ * Department of ECEE, University of Hertfordshire, Hatfield AL10 9AB, UK; Email: y.sun@herts.ac.uk
}

\begin{abstract}
A fully differential R-MOSFET-C fourthorder Bessel active lowpass filter employing passive resistors and current-steering MOS transistors as a variable resistor is proposed. The implementation relies on the tunability of the current-steering MOS transistors operating in triode region which counteract the concert deviation of resistance in integrated circuit manufacturing technology in order that the group delay of the Bessel active filter can be realised accurately. A 0.75 us group delay $520 \mathrm{kHz}$ fourth-order Bessel lowpass filter based on a passive doubly terminated RLC prototype was designed and fabricated using $3.3 \mathrm{~V}$ power supply and 0.5 um CMOS technology. Chip test results demonstrate better than $65 \mathrm{~dB}$ THD with a $100-\mathrm{kHz}, 1.65-\mathrm{Vpp}$ input signal, frequency tuning range of more than ten decade from $0.6 \mathrm{kHz}$ to $550 \mathrm{kHz}$, chip area of $0.32 \mathrm{~mm}^{2}$ and power consumption of $13.3 \mathrm{~mW}$.
\end{abstract}

\section{INTRODUCTION}

Design of integrated continuous-time active filters has become an active research area [1-9]. CT filters have been widely and successfully used in modern communication systems such as the ADSL system and GSM baseband I/O port circuit, etc $[5,6]$. Generally, design of active filters may employ the Opamp-RC, Gm-C and MOSFET-C structures. However, the cut-off frequency of the OpampRC filter is not easy to tune and thus difficult to achieve high accuracy, because there exists concert deviation of resistances in IC manufacturing technology. The cut-off frequency of the $\mathrm{Gm}-\mathrm{C}$ filter can be tunable through adjusting the $\mathrm{Gm}$ value of the transconductor. However, due to the open-loop operation nature, $\mathrm{Gm}-\mathrm{C}$ filters usually perform poorly as far as linearity is concerned. To achieve both high accuracy and large dynamic range,

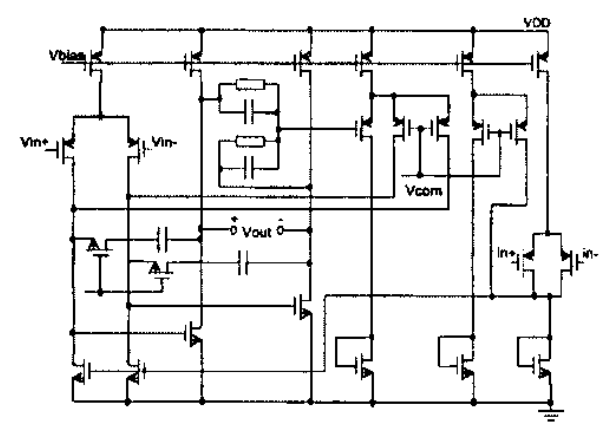

Fig. 1 Two-stage fully differential op-amp
MOSFET-C filters seem the choice.

To obtain a very accurate group delay for the GSM baseband I/O port IC application, a Bessel: filter with frequency accuracy of $\pm 1 \%$ is required. This can be achieved by using R-MOSFET-C technique. Adjusting the gate voltages of those MOS transistors in triode region, we can change the active MOS transistor resistances to counteract the concert deviation of passive resistances in IC manufacturing technology.

The CMOS R-MOSFET-C fourth-order Bessel filter to be presented in this paper has been designed for a mobile phone application operating with a minimum power supply of $3.3 \mathrm{~V}$. The filter is used in the transmitter as a smoothing filter for I and Q modulated signals coming from digital-to-analog converters. Maximum total harmonic distortion (THD) of $-65 \mathrm{~dB}$ is required for a 1.65 Vpp input signal. The filter must have a small dc gain variation and a constant group delay in order not to influence the GMSK modulated signal spectrum. Excellent gain and phase matching between the I and Q channels are also required to avoid additional sideband frequency components. Details are given in the following.

\section{OP AMP AND INTEGRATOR}

\section{A. Fully differential op amp}

A two stage fully differential operational amplifier with approximately $78.5-\mathrm{dB}$ dc gain, $28-\mathrm{MHz}$ unity-gain bandwidth, 87-degree phase margin, and 18 -dB gain margin when the load is $2 \mathrm{pF}$ (from simulation) is used in the filter design. A high-gain amplifier was chosen to minimize the distortion of the filter. The op amp with a frequency compensation network (standard Miller compensation) is shown in Fig.1.

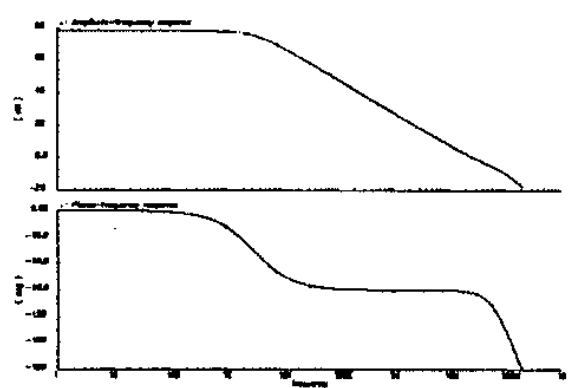

Fig. 2 Op-amp amplitude and phase frequency responses 
The op amp aiso has a common-mode feedback circuit which stabilizes the DC operating point. It operates at a single $3.3 \mathrm{~V}$ power supply and the common-mode voltage is $1.65 \mathrm{~V}$. Simulation results are given in Fig.2, showing the open-loop amplitude and phase responses of the fully differential op amp.

\section{B. R-MOSFET-C integrator}

A differential balanced variable resistor made of four identical MOS transistors MI, M2, M3, and M4 biased in triode region combined with two passive resistors, is shown in Fig.3. The resistance (inherently conductance) of the current steering portion (MOSFETS) is set by control voltage $\mathrm{Vc}$ and the whole R-MOSFET stage has an equivalent resistance of:

$$
R_{e q}=\frac{A}{G_{t, 2}-G_{3,4}}=\frac{A}{K V C}
$$

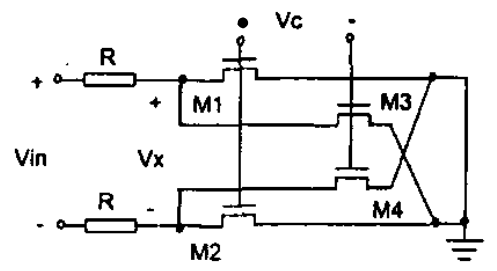

\section{Fig.3 R-MOSFET resistor}

The combined R-MOSFET stage in Fig. 3 in conjunction with an op amp with feedback capacitors forms a low distortion R-MOSFET-C integrator, as shown in Fig.4. The integrator displays a linearly controlled unity-gain frequency, given by

$$
\omega_{\text {unity }}=\frac{G_{1,2}-G_{3,4}}{A C}=\frac{K V C}{A C}
$$

\section{ACTIVE FILTER STRUCTURE}

Using the above R-MOSFET-C integrator, a fourth order lowpass filter was designed. Through tuning the where the voltage scale factor $A$ is defined by

$$
\begin{gathered}
A=\frac{V_{i}}{V_{x}}=1+2 \bar{G} R \\
G_{i}=K\left(V_{G S i}-V_{t h l}\right) \\
K=\mu_{n} C_{O X} \frac{W}{L}
\end{gathered}
$$

The average conductance of M1, M2, M3, and M4 is

$$
\bar{G}=\frac{\left(G_{1,2}+G_{3,4}\right)}{2}
$$

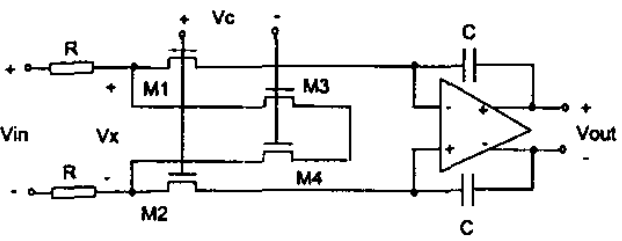

Fig.4 Fully differential R-MOSFET-C integrator

control voltage $\mathrm{Vc}$, the frequency accuracy of the filter was achieved within $\pm 1 \%$. Therefore, the goal of accurately designing the filter group delay was realized.

To obtain a lowpass frequency response with minimal sensitivities to individual component values, the filter topology is derived from a doubly terminated passive RLC lowpass prototype as shown in Fig.5(a). The associated signal flow graph (SFG), shown in Fig.5(b), is obtained by writing down the state equations for the four reactive components. Notice that the SFG contains only lossless integrators, lossy integrators and summers. 




(a)

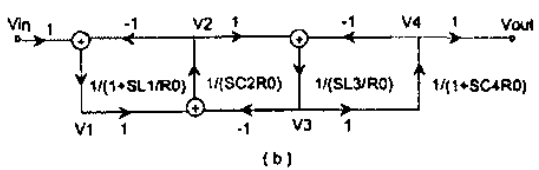

(b)

Fig. 5 Normalized fourth-order lowpass filter

(a) LCR prototype and (b) signal flow graph

From Fig.5, the state equations can be obtained as [1]:

$$
\begin{gathered}
V_{1}=\frac{1}{1+S \frac{L_{1}}{R_{0}}}\left(V_{\text {in }}-V_{2}\right) \\
V_{2}=\frac{1}{S C_{2} R_{0}}\left(V_{1}-V_{3}\right) \\
V_{3}=\frac{1}{S \frac{L_{3}}{R_{0}}}\left(V_{2}-V_{4}\right) \\
V_{\text {out }}=V_{4}=\frac{V_{3}}{1+S C_{4} R_{0}}
\end{gathered}
$$

According to the signal flow graph, the fourth-order fully differential active lowpass filter based on ideal integrators and lossy integrators can be obtained as shown in Fig.6. The integrators are composed of R-MOSFET-C devices and op amps.

The state equations of Fig. 6 can be derived as:

$$
\begin{gathered}
V_{1}=\frac{1}{1+S C_{1} R}\left(V_{\text {in }}-V_{2}\right) \\
V_{2}=\frac{1}{S C_{2} R}\left(V_{1}-V_{3}\right) \\
V_{3}=\frac{1}{S C_{3} R}\left(V_{2}-V_{4}\right) \\
V_{\text {out }}=V_{4}=\frac{1}{1+S C_{4} R} V_{3}
\end{gathered}
$$

\section{DESIGN OF BESSEL FILTER}

The transfer function of $0-d B$ passband gain group delay fourth-order Bessel filter is given by

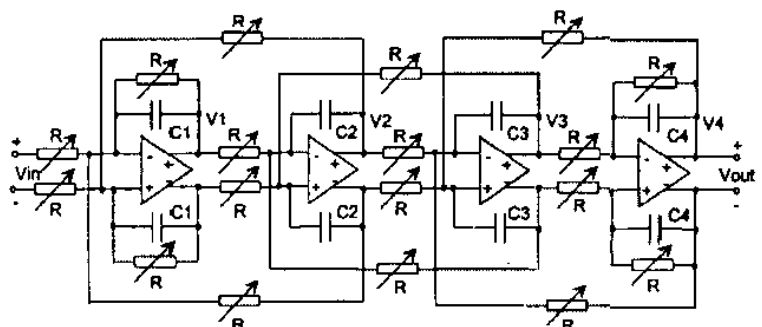

ig. 6 Fourth-order fully differential active lowpass filter

$$
H(S)=\frac{105}{S^{4}-10 S^{3}+45 S^{2}-105 S+105}
$$

which has a normalized cutoff angular frequency of $\omega=2.114$.

To realize the group delay $\mathrm{T}_{0}=0.75 \mathrm{us}$, we calculate the $L C R$ values of the prototype filter as:

$\mathrm{L}_{1}=5.32 \times 10^{-2} \mathrm{H}, \mathrm{C}_{2}=3.47 \times 10^{-12} \mathrm{~F}$,

$\mathrm{L}_{3}=2.17 \times 10^{-2} \mathrm{H}, \mathrm{C}_{4}=0.75 \times 10^{-12} \mathrm{~F}, \mathrm{R}_{0}=1 \times 10^{5} \Omega$

The $\mathrm{RC}$ values of the active filter are determined as:

$\mathrm{C}_{1}=5.32 \times 10^{-12} \mathrm{~F}, \mathrm{C}_{2}=3.47 \times 10^{-12} \mathrm{~F}$,

$\mathrm{C}_{3}=2.17 \times 10^{\circ}$

${ }^{12} \mathrm{~F}, \mathrm{C}_{4}=0.75 \times 10^{-12} \mathrm{~F}, \mathrm{R}=10^{5} \Omega$.

As discussed in Section II with a particular focus on the R-MOSFET circuit, each of the passive resistors in the active RC filter needs to be replaced by a variable RMOSFET combined resistor, resulting in a highly linear RMOSFET-C implementation, where in the R-MOSFET combination, $\mathrm{R}$ summing the equivalent MOS transistor resistance is equal to the original single resistance, and the capacitance value stays unchanged. The tunability of the MOSFET resistor makes the accurate realization of the filter group delay possible.

\section{EXPERIMENTAL RESULTS}

The fully differential Bessel lowpass filter with group delay of 0.75 us and cutoff frequency of $520 \mathrm{kHz}$ has been fabricated using $3.3 \mathrm{~V}$ power supply and $0.5 \mathrm{um}$ CMOS technology. The active chip area of the filter is about $0.32 \mathrm{~mm}^{2}$ which is much smaller than that in [6] and the power consumption of the filter is about $13.3 \mathrm{~mW}$.

Fig. 7 shows the measured group delay response of the filter which fits well with the ideal response. The measured frequency responses which have a tuning range of almost ten decade from $0.6 \mathrm{kHz}$ to $550 \mathrm{kHz}$, are given in Fig.8. The tuning range is much wider than that in [6]. The measured in-band gain variation for each tuned frequency 
is about $0.01 \mathrm{~dB}$, group delay smaller than $25 \mathrm{~ns}$, THD for a $1.65 \mathrm{Vpp}$ input sinewave of $100 \mathrm{kHz}$ less than $-65 \mathrm{~dB}$, and

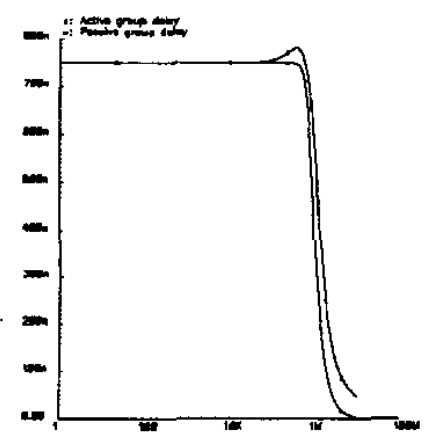

Fig. 7 Ideal and practical group delay responses of Bessel filter the common-mode rejection ratio better than $-90 \mathrm{~dB}$. Measured performances are summarized in Table I

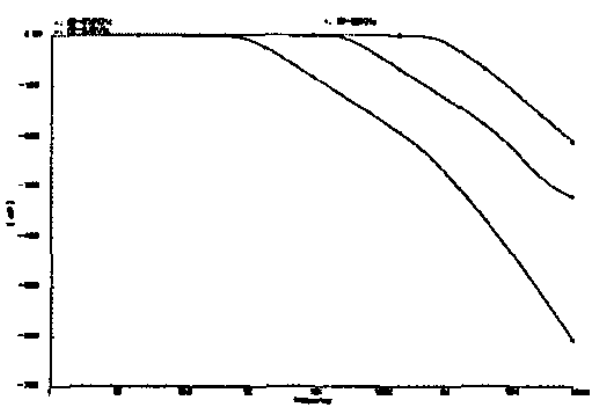

Fig. 8 Measured filter magnitude responses with cutoff frequencies at $0.6 \mathrm{kHz}, 20 \mathrm{kHz}$, and $550 \mathrm{kHz}$

Table 1 Summary of measured performances of Bessel filter

$-3 \mathrm{~dB}$ frequency
Frequency tuning range
Passband gain variation
Group delay deviation $(<500 \mathrm{kHz})$
THD(@100kHz and Vin $<2 \mathrm{Vpp})$
CMRR
PSRR(DC), positive supply
PSRR(DC), negative supply
Chip area
Power consumption

\section{CONCLUSIONS}

This paper has described a fully differential $\mathrm{R}$ MOSFET-C lowpass Bessel filter whose group delay can be designed accurately through the tuning gate voltage of MOS transistors which operate in triode region. A fourthorder 0.75 us group delay $520 \mathrm{kHz}$ Bessel lowpass filter was designed by simulation of a passive RLC prototype and tested using a single $3.3 \mathrm{~V}$ supply voltage and $0.5 \mathrm{um}$ CMOS technology. Measured results have shown that the filter has THD of only $-65 \mathrm{~dB}$ for a $100 \mathrm{kHz} 1.65 \mathrm{Vpp}$ input signal, wide frequency tuning range from $0.6 \mathrm{kHz}$ to $550 \mathrm{kHz}$, group delay deviation in passband of smaller than $22 \mathrm{~ns}$, chip area of $0.32 \mathrm{~mm}^{2}$ and power consumption of $13.3 \mathrm{~mW}$.

\section{REFERENCES}

[1] T. Deliyannis, Y. Sun and J. K. Filder, Continuous-time Active Filter Design, CRC Press, USA, 1999.

[2] G Groenewold, "Low-power MOSFET-C $120 \mathrm{MHz}$ Bessel allpass filter with extended tuning range," IEE Proceedings: Circuits Devices Syst, vol.147, No.1, 2000.
$520 \mathrm{kHz}$

$0.6 \mathrm{kHz} \sim 550 \mathrm{kHz}$

$<0.01 \mathrm{~dB}$

$<25 \mathrm{~ns}$

$<-55 \mathrm{~dB}$

$>60 \mathrm{~dB}$

$80 \mathrm{~dB}$

$90 \mathrm{~dB}$

$0.32 \mathrm{~mm}^{2}$

$13.3 \mathrm{~mW}$

[3] H.P. Schmid and G S. Moschytz, "Active-MOSFET-C single amplifier biquadratic filters for video frequencies, " IEE Proc. CDS, vol. 147, No. 1, 2000.

[4] C. Hill, Y. Sun and H. Su, "Low power CMOS filter for video frequencies", IEE Colloq. on LP IC Design, London, 2000.

[5] Z. Y. Chang, D. Haspelagh, and J. Verfaillie, "A highly linear CMOS Gm-C bandpass filter with on-chip frequency tuning," IEEE JSSC, vol.32, March 1997.

[6] F. Yang and C. C. Enz, "A low-distortion BICMOS seventhorder Bessel filter operating at $2.5 \mathrm{~V}$ supply," IEEE J. SolidState Circuits, vol.31, March 1996.

[7] S. Lindfors, J. Jussila, et al, "A 3-V continuous-time filter with on-chip tuning for IS-95," IEEE JSSC, vol. 34, 1999.

[8] H. Yamazaki, K. Oishi, and K. Gotoh, "An accurate center frequency tuning scheme for $450-\mathrm{kHz}$ CMOS $\mathrm{Gm}-\mathrm{C}$ bandpass filters," IEEE JSSC, vol.34, Dec 1999.

[9] Z.Y. Chang, et al, "A CMOS analog front-end circuit for an FDM-based ADSL system," IEEE JSSC, vol.30, Dec 1995. 